\section{PET Amyloid Analyses}

\author{
Kirk A. Frey and Robert A. Koeppe \\ University of Michigan Hospitals, Ann Arbor, Michigan
}

$\mathbf{I}_{n}$ this issue of The Journal of Nuclear Medicine, Rowe et al. describe application of the centiloid methodology for scaling and analysis of studies performed with ${ }^{18} \mathrm{~F}-\mathrm{NAV} 4694$ for characterizing cerebral amyloid deposition in clinical research (1). This is one of several evolving approaches to analysis of amyloidtargeting radiotracers that have emerged recently. The centiloid approach describes a standard method of analyzing ${ }^{11} \mathrm{C}$-Pittsburgh compound $\mathrm{B}\left({ }^{11} \mathrm{C}-\mathrm{PiB}\right) \mathrm{PET}$ data and then provides a method for scaling of any nonstandard method of ${ }^{11} \mathrm{C}-\mathrm{PiB}$ imaging or of image data from different amyloid radiotracer probes (e.g., ${ }^{18} \mathrm{~F}$-florbetapir, ${ }^{18} \mathrm{~F}$-flutemetamol, ${ }^{18} \mathrm{~F}$-florbetaben, and, now, $\left.{ }^{18} \mathrm{~F}-\mathrm{NAV} 4694\right)$ to the same scale, the centiloid scale (2). The centiloid method thus provides a common quantitative scale applicable across studies using differing imaging methodologies and tracers. It is anticipated that this approach will facilitate aggregation of experimental findings across laboratories and across radiotracers.

\section{See page 1233}

The initial step in centiloid scaling, as described previously (2), provided the conversion of a standard ${ }^{11} \mathrm{C}-\mathrm{PiB}$ SUV ratio (SUVR) measure to centiloids. This involved selecting groups of young healthy subjects and of typical Alzheimer disease subjects and analyzing ${ }^{11} \mathrm{C}-\mathrm{PiB}$ PET activity data from 50 to $70 \mathrm{~min}$ after intravenous-bolus tracer injection. After anatomic transformation to a reference atlas orientation and space, a standard target volume (predominantly cerebral neocortex) and a standard reference volume (whole cerebellum) are used to calculate a single SUVR per subject. A linear transformation is then used to scale the mean of the young controls to a centiloid value of 0 and the mean of the Alzheimer subjects to 100. Conversion of PET data from another tracer then requires scanning the same subjects again and performing regression between the standard, ${ }^{11} \mathrm{C}-\mathrm{PiB}$, and the second tracer.

Received Mar. 7, 2016; revision accepted Mar. 11, 2016.

For correspondence or reprints contact: Kirk A. Frey, University of Michigan Hospitals, Room B1 G505 UH, 1500 E. Medical Center Dr., Ann Arbor, MI 48109-0028.

E-mail: kfrey@umich.edu

Published online Apr. 14, 2016.

COPYRIGHT (C) 2016 by the Society of Nuclear Medicine and Molecular Imaging, Inc.

DOI: $10.2967 /$ jnumed.116.173989
The centiloid method may prove useful in several scenarios, allowing standardization of results across differing amyloid radiotracers, brain reference regions, and experimental imaging protocols. The current report (1) is an example of the first scenario. Using the standard target and reference VOIs (2), SUVRs were generated for both ${ }^{11} \mathrm{C}-\mathrm{PiB}$ and ${ }^{18} \mathrm{~F}-\mathrm{NAV} 4694$ in each subject, and a regression was performed relating the new radiotracer SUVR to the ${ }^{11} \mathrm{C}-\mathrm{PiB}$ SUVR. Once the regression parameters were determined, ${ }^{18} \mathrm{~F}-\mathrm{NAV} 4694$ SUVR measures could be converted directly into centiloid values. The conversion to centiloids is based on a single scale factor per amyloid scan and is not brain region-dependent.

A second aspect of amyloid image processing that has recently been discussed concerns the selection of a brain reference region for scaling of the image intensity values $(3,4)$. Several groups of investigators have determined that the use of the traditional cerebellar cortical reference region may be disadvantaged by a series of factors, including low signal activity and positioning near the axial edge of typical brain PET image acquisitions, that results in poor statistical and underlying kinetic properties of the reference region. The application of centiloids can allow the use of different reference tissues while maintaining the ability to report amyloid binding on a standardized scale. The recommendation arising from these studies includes the use of supratentorial cerebral white matter as at least a portion of the scaling reference value. This results in (seemingly) more plausible longitudinal scan results, with surprisingly limited correlations between values in the standard cerebellar cortex and the white matter-containing reference region $(3,4)$.

Finally, there is ongoing debate over the experimental design most appropriate to quantification of amyloid tracer binding; specifically, the use of dynamic imaging and kinetic determinations of tracer distribution volume versus the technically simpler late-static-imaging determination of SUVR. Again, investigators have reported more robust and apparently less variable results from longitudinal analyses of distribution volume ratio than of SUVRs (5).

All three of these recent technical embellishments are likely to have a significant, positive impact on the precision and accuracy of quantitative amyloid imaging. The new methodologies will predictably be most important to studies of interval change in amyloid deposition and in the relationships between amyloid measures and clinical correlates of disease. The clinical use of amyloid imaging to determine the presence (positive) or absence (negative) of Alzheimer disease-range fibrillary amyloid deposition in individual patients is unlikely to require these refinements, although there is at least one report suggesting that qualitative review of amyloid tracer imaging is more reproducible with distribution volume ratio than with SUVR approaches (6). For 
routine clinical interpretation of amyloid status, clinicians should refer to the interpretative criteria approved in package inserts by the U.S. Food and Drug Administration for each agent.

\section{REFERENCES}

1. Rowe CC, Jones G, Doré V, et al. Standardized expression of ${ }^{18} \mathrm{~F}-\mathrm{NAV} 4694$ and ${ }^{11} \mathrm{C}$-PiB $\beta$-amyloid PET results with the centiloid scale. J Nucl Med. 2016;57: $1233-1237$.

2. Klunk WE, Koeppe RA, Price JC, et al. The Centiloid Project: standardizing quantitative amyloid plaque estimation by PET. Alzheimers Dement. 2015;11:1-15.
3. Chen K, Roontiva A, Thiyyagura P, et al., for the Alzheimer's Disease Neuroimaging Initiative. Improved power for characterizing longitudinal amyloid- $\beta$ PET changes and evaluating amyloid-modifying treatments with a cerebral white matter reference region. J Nucl Med. 2015;56:560-566.

4. Landau SM, Fero A, Baker SL, et al. Measurement of longitudinal $\beta$-amyloid change with ${ }^{18} \mathrm{~F}$-florbetapir PET and standardized uptake value ratios. J Nucl Med. 2015;56:567-574

5. van Berckel BNM, Ossenkoppele R, Tolboom N, et al. Longitudinal amyloid imaging using ${ }^{11} \mathrm{C}-\mathrm{PiB}$ : methodologic considerations. J Nucl Med. 2013;54: $1570-1576$.

6. Zwan MD, Ossenkoppele R, Tolboom N, et al. Comparison of simplified parametric methods for visual interpretation of ${ }^{11} \mathrm{C}$-Pittsburgh compound-B PET images. $\mathrm{J} \mathrm{Nucl}$ Med. 2014;55:1305-1307. 